\title{
Philonsorbonne
}

6 | 2012

Année 201 1-2012

\section{La notion de bon sens dans la philosophie d'Henri Bergson}

\section{Clarisse ZOULIM}

\section{(2) OpenEdition \\ 1 Journals}

Édition électronique

URL : https://journals.openedition.org/philonsorbonne/394

DOI : 10.4000/philonsorbonne.394

ISSN : $2270-7336$

Éditeur

Publications de la Sorbonne

\section{Édition imprimée}

Date de publication : 15 juillet 2012

Pagination : 83-96

ISBN : 978-2-85944-710-6

ISSN : 1255-183X

Référence électronique

Clarisse ZOULIM, "La notion de bon sens dans la philosophie d'Henri Bergson », Philonsorbonne [En ligne], 6 | 2012, mis en ligne le 04 février 2013, consulté le 08 juin 2021. URL : http://

journals.openedition.org/philonsorbonne/394; DOI : https://doi.org/10.4000/philonsorbonne.394

(c) Tous droits réservés 


\section{La notion de bon sens \\ dans la philosophie d'Henri Bergson}

Clarisse Zoulim

La notion de bon sens, mentionnée explicitement dans la plupart des ouvrages de Bergson, depuis Matière et Mémoire jusqu'aux Deux sources de la morale et de la religion, ne constitue pas à proprement parler un concept central de la pensée bergsonienne, ne serait-ce qu'en vertu du peu d'importance qui lui est, quantitativement, accordé. Cela dit, après l'avoir examinée pour la première fois en détail dans le discours de remise des prix du concours général prononcé le 30 juillet 1895 et intitulé Le bon sens et les études classiques, Bergson n'aura de cesse de revenir sur la définition de cette notion et sur la description de la réalité à laquelle elle renvoie. L'étude de cette notion a donné lieu à des interprétations très différentes chez les commentateurs et il semble que cela soit dû à deux facteurs : premièrement, cherchant pour la plupart à considérer la notion de bon sens comme un bloc homogène malgré certaines contradictions manifestes entre les différents ouvrages, ils n'ont pas assez pris en compte les évolutions et modifications qui ont marqué le trajet de la notion de bon sens dans le corpus bergsonien depuis le discours de 1895. Deuxièmement, il semble que la plupart d'entre eux ait accordé une trop faible importance à la distinction que Bergson opère dans Les deux sources de la morale et de la religion, entre un bon sens ordinaire et un bon sens qu'il désigne comme «bon sens supérieur ». Ainsi, sans céder à la tentation de produire « une exposition plus systématique de la doctrine et aussi l'apparence d'une clarté supérieure ${ }^{1}$, nous souhaiterions mettre en avant le fait que l'examen attentif des variations subies par la notion de bon sens permet de mettre en lumière le trajet de la pensée de Bergson elle-même en ce qui concerne la question de l'action juste (et plus largement, du champ de l'activité morale). 
En effet on peut remarquer, dès le discours de 1895, l'entrecroisement de deux lignes dans la définition du bon sens : il est posé à la fois comme une santé mentale, comme le bon fonctionnement de l'esprit qui permet son adaptation souple, sa disponibilité à l'égard d'une réalité se renouvelant sans cesse, mais aussi comme un «instrument, avant tout, de progrès social $»^{2}$, mu par ce que Bergson désigne comme «l'esprit de justice». Cette binarité est reprise dans la lettre à $\mathrm{O}$. Gérard, préambule du discours dans l'édition des Mélanges : le bon sens est «la faculté de raisonner juste, non seulement sur ses propres affaires, mais encore et surtout sur celles du pays $»^{3}$, le bon sens étant caractérisé à la fois par la justesse de son adaptation et la justice de son action. Or, il apparaît très vite que les ouvrages immédiatement ultérieurs ne reprennent et n'approfondissent que la première dimension de cette définition, faisant du bon sens une forme originale de santé mentale, pensée comme effort et comme équilibre. À partir du Rire puis dans l'Évolution créatrice, cette caractérisation du bon sens comme «vie psychologique normale ${ }^{4}$ s'enrichit d'une réflexion sur la dimension proprement sociale de la réalité humaine : le bon sens, sens social défini comme souple disponibilité à l'égard de la fluidité des échanges humains, intègre à présent un certain nombre de normes et de paramètres sociaux dans son fonctionnement. C'est cette dépendance du normal psychologique à l'égard des normes sociales que nous envisagerons dans un premier temps.

C'est seulement dans Les deux sources de la morale et de la religion, sur la base d'une réflexion sociologique renouvelée qui distingue à présent deux types de sociétés (c'est-à-dire, deux types de rapports entre individus et normes sociales) et deux types de justices, que le bon sens retrouve la dimension morale (comme facteur de progrès social produisant des actions justes) que le discours lui prêtait. Mais ce retour ne se fait qu'au prix de la distinction entre un bon sens supérieur et un bon sens ordinaire qui permet, certes, de résoudre les difficultés posées par le discours de 1895, en expliquant par le «bon sens supérieur» la possibilité d'une action créatrice de normes, mais qui semble cependant remettre en question la définition du normal psychologique donnée précédemment, ou qui suggère tout du moins l'existence d'un conflit latent entre la morale et le normal dans la pensée bergsonienne. Nous examinerons donc en second lieu cette distinction capitale, qui met d'autant plus en jeu le rapport de la définition du «normal » à la problématique morale selon laquelle l'individu moral, le mystique, est un individu anormal en au moins deux sens, qui se trouvaient au cœur du bon sens ordinaire : d'une part, en échappant à l'égoïsme et la loi du talion qui prévalent dans la société close, il ne s'adapte plus aux normes sociales mais les modifie; et d'autre part, sa singularité s'exprime par

2. Ibid., p. 364.

3. Ibid., p. 360.

4. H. Bergson, L'énergie spirituelle, op. cit., p. 893 : «Cela, c'est veiller, c'est vivre la vie psychologique normale, c'est lutter, c'est vouloir ». 
des manifestations pathologiques, qu'elles soient appelées délire mystique ou «perceptions anormales ».

Un bref examen du bon sens tel qu'il est caractérisé depuis Matière et Mémoire jusqu'à l'Évolution créatrice permet de voir combien le normal, la santé mentale qu'il incarne, est déterminé par les exigences vitales et sociales : à partir de Matière et Mémoire, le bon sens apparaît en effet à la fois comme un équilibre psychologique entre deux tendances ennemies de l'action, la tendance corporelle à l'automatisme et la tendance spirituelle au rêve $^{5}$, et comme effort d'attention à la réalité ${ }^{6}$. Il est en effet présenté comme une tension visant à tenir à égale distance les souvenirs issus des deux pôles opposés du moi que sont la mémoire corporelle et la mémoire pure et qui cherchent à s'insérer dans la perception présente. Agissant en amont du travail discriminant de la conscience en « laiss[ant] tous les souvenirs dans le rang ${ }^{7}$, le bon sens déblaie le terrain à partir duquel celle-ci sélectionnera, en s'appuyant sur les formes motrices, la schématisation des souvenirs purs, les lois du rappel et la condition générale de ressemblance, le souvenir apte à s'intégrer profitablement à la perception présente pour l'informer. En tant que «sens pratique», il est gage de souplesse dans la prise en compte de la réalité présente et muselle la tendance à agir mécaniquement, sous l'impulsion de la reconnaissance automatique qu'a la mémoire habitude des «idées générales ${ }^{8}$ dans la perception. En tant que «sens du réel ${ }^{9}$, il est lié à la veille et est un effort de tension contre l'évasement, la diffusion des souvenirs purs qui caractérise la dérive pathologique de Don Quichotte, en qui la mémoire pure a pris l'ascendant et qui, au lieu d'utiliser ses souvenirs pour percevoir de manière adaptée, «se sert au contraire de ce qu'il perçoit pour donner un corps au souvenir préféré ${ }^{10}$. En tant qu' « oubli réglementé et durable, $[\ldots]$ art de liquider son passé ${ }^{11}$, le bon sens s'insère dans une conception du normal fondée sur l'idée que la santé mentale résulte d'un effort de tension qui s'écarte donc par degrés du pathologique (qui peut résulter alors d'une forme de paresse psychologique telle qu'elle a été identifiée par Pierre Janet chez les psychasthéniques ${ }^{12}$ ), telle qu'elle

5. Id., Matière et mémoire, Euvres complètes, op. cit., p. 294 : «Entre ces deux extrêmes [l'impulsif et le rêveur] se place l'heureuse disposition d'une mémoire assez docile pour suivre avec précision les contours de la situation présente, mais assez énergique pour résister à tout autre appel. Le bon sens, ou sens pratique, n'est vraisemblablement pas autre chose ».

6. Il est, dans Le Rire, «continuité mouvante de notre attention à la vie », Euvres, op. cit., p. 475.

7. Ibid., p. 475.

8. Matière et Mémoire, op. cit., p. 296-302.

9. Mélanges, op. cit., p. 620.

10. Le Rire, op. cit., p. 476.

11. V. Jankélévitch, Henri Bergson, Paris, PUF, 2008, p. 127-128.

12. H. Bergson, Mélanges, op. cit., p. 620 : «Tous ne sont pas capables de cette vie de travail, tous n'ont pas également le sens du réel, le bon sens ». Pierre Janet remarque chez les psychasthéniques un «caractère fondamental, difficile à expliquer, [...] le caractère de la paresse » (La force et la faiblesse psychologiques, Paris, éditions médicales Norbert Maloine, 1932, p. 275). 
s'exprime surtout à partir de l'Energie spirituelle ${ }^{13}$. Par cet effort de tension, l'individu se rend capable de fournir une réponse ajustée aux circonstances et à ce que requiert la situation, le bon sens est donc lié aux exigences vitales qui déterminent l'adaptation de l'individu, la survie individuelle.

Même si Bergson distinguait déjà dans le discours de 1895 le milieu naturel, domaine d'action des sens, du « milieu social», domaine d'action du bon sens, c'est seulement à partir du Rire qu'il entame une analyse de la dimension proprement sociale de l'existence, et c'est aussi dans cet ouvrage que le bon sens, dont l'opposé est désormais « le comique [qui] exprime avant tout une certaine inadaptation particulière de la personne à la société ${ }^{14}$, se précise sous l'aspect d'une adaptation au réel dans sa dimension sociale. Cet aspect de la notion se retrouve, très fortement accentué, dans Les deux sources, où le bon sens est non seulement décrit comme «sens social » mais encore caractérisé comme «inné à l'homme normal $»^{15}$ : cette innéité qui équivaut à la « préfiguration de la société dans l'individu ${ }^{16}$, s'explique par la nécessité pour la nature de «donner à l'homme des directives, au moins générales, pour la coordination de sa conduite à celle de ses semblables ${ }^{17}$. Aussi, s'il n'y a «nul doute que notre structure psychologique ne tienne à la nécessité de conserver et de développer la vie individuelle et sociale ${ }^{18}$, cela signifie que le bon sens embrasse dans sa compréhension de la situation présente la compréhension des normes qui régissent l'agir en société et, par conséquent, que celles-ci s'intègrent à ce qui fait chez Bergson la santé mentale. Ainsi, le «délire d'interprétation » qui se caractérise par une incapacité à comprendre le comportement d'autrui, résulterait, selon Les deux sources, d'un défaut de bon sens qui s'expliquerait in fine par une «insuffisance psychique [...] congénitale $\gg^{19}$. Le bon sens prend donc ici les traits d'une prédisposition à s'adapter avec fluidité aux impératifs sociaux, c'est-à-dire d'une souplesse adaptative prédéterminée, point qui marque sans conteste une originalité de la pensée biologique et sociologique bergsonienne.

Déjà, à travers les mutations subies entre l'Essai et le Rire par la notion de « caractère » (qui devient dans le Rire « ce qu'il y a de tout fait dans notre personne, ce qui est en nous à l'état de mécanisme une fois monté, capable de fonctionner automatiquement $\gg^{20}$ ), apparaît une tension entre l'idée,

13. Dans Matière et Mémoire, Bergson semble encore n'attribuer à l'aliénation que des causes biologiques, à savoir la «perturbation des relations sensori-motrices établies dans l'organisme » (p. 313).

14. Le Rire, op cit., p. 450.

15. Les deux sources de la morale et de la religion, p. 1065.

16. Guy Lafrance, La philosophie sociale de Bergson, sources et interprétation, Ottawa, éditions de l'université d'Ottawa, 1974, p. 115 et suiv.

17. H. Bergson, Les deux sources de la morale et de la religion, op. cit., p. 1065.

18. Ibid., p. 1065.

19. Ibid. p. 1065.

20. Le Rire, op. cit., p. 457-458. 
exprimée dans le discours, que le bon sens aurait partie liée avec la liberté du moi telle qu'elle était évoquée par l'Essai, et sa définition comme capacité de souple adaptation sociale dans le Rire. En effet, il faut remarquer non seulement que cette adaptation à la mouvance de la réalité sociale aurait peut-être été jugée automatique du point de vue de l'Essai en tant qu'émanant des couches superficielles du moi (comme dans le fameux exemple du réveil), mais encore que la critique de l'automatisme dans le Rire effectue un renversement puisqu'elle n'est plus tournée contre ce qu'une action peut avoir d'impersonnel mais bien contre ce qu'elle peut avoir de trop personnel au détriment d'une prise en compte des autres : « est comique le personnage qui suit automatiquement son chemin sans se soucier de prendre contact avec les autres ${ }^{21}$ et qui «néglige de regarder autour de soi $»^{22}$. S'il est possible de considérer, pour résoudre cette tension, que le bon sens a partie liée avec la transition du moi profond vers le moi superficiel, nécessaire à l'insertion de l'esprit dans la matière en amont de l'insertion dans une situation sociale, il n'empêche qu'elle apparaîtra à nouveau dans la distinction entre bon sens ordinaire et bon sens supérieur, sous la forme d'une tension entre une impulsion venue du moi profond et s'imposant à l'extérieur et une impulsion venue de l'extérieur s'imposant, en le solidifiant, au moi profond.

Si la description du bon sens ordinaire a mis hors-jeu la problématique de la justice et de l'action morale en charge du progrès social telle qu'elle était exprimée dans le discours de 1895 (et qui rappelait le lien établi par Descartes entre bon sens et recherche d'une vérité pratique $\left.{ }^{23}\right)$, c'est

21. Ibid., p. 451.

22. Ibid., p. 457.

23. Bergson fait référence de manière explicite à la conception cartésienne du bon sens dans le discours de 1895, il est même possible d'envisager que c'est en hommage au bon sens analysé par Descartes dans le Discours de la méthode que Bergson a conservé le terme «bon sens » pour désigner l'équilibre psychique qu'il décrit. Les références à la problématique cartésienne du bon sens abondent dans le discours de 1895 : le concept d'attention peut suggérer un arrière plan cartésien, et Bergson y évoque l'urgence de l'action en des termes qui rappellent ceux de Descartes pour qui « les actions de la vie ne souffrent aucun délai ». Dans le discours de 1895, Bergson désigne le bon sens comme une «ignorance consciente d'ellemême », termes qu'il répétera à l'identique dans son hommage à Descartes en 1937, lorsqu'il fait de celui-ci l'auteur de l'idée selon laquelle «la vraie connaissance a moins de rapport avec une information superficiellement encyclopédique qu'avec une ignorance consciente d'elle-même ». Dans Le bon sens et les études classiques, Bergson ne nie pas directement que le bon sens soit «la chose du monde la mieux partagée », mais il affirme que, si le bon sens est une faculté «inné[e] et universel[le]», celle-ci est empêchée par certains obstacles, notamment les préjugés et la distraction avec laquelle l'individu les reçoit : l'universalité en droit du bon sens s'accorde bien avec l'idée que le bon sens représente une sorte de santé mentale. Mais, à la différence du bon sens cartésien, le bon sens bergsonien ne nécessite pas l'adoption d'une méthode : l'adaptation à la réalité qui résulte du bon sens est spontanée, l'action qui en est issue tranche, brise le cercle, puisqu'elle est puissance d'invention de solutions. En outre, il n'est pas à proprement parler une faculté, que l'on pourrait rabattre soit sur l'intelligence, soit sur l'intuition, mais bien plutôt un état d'équilibre entre facultés, tempérant l'action de chacune en vue d'une bonne adaptation. 
précisément d'une part parce que le principe selon lequel le vital engendrerait le social qui engendrerait à son tour le normal, que Bergson pose au fondement même de sa définition de la santé mentale, contribue à substituer à la problématique de la justice de l'action celle de la justesse de l'adaptation. D'autre part, cela est incontestablement lié à l'examen du fonctionnement de la vie sociale auquel Bergson se livre dans Le Rire puis dans Les deux sources, et qui lui a permis d'affirmer dès le premier de ces ouvrages qu' «être en règle avec la stricte morale » n'a rien à voir avec le fait de «se mettre en règle avec la société » ${ }^{24}$ : le bon sens, devenu faculté d'adaptation souple au réel social et d'insertion bien ajustée dans le tissu humain, apparaît définitivement lié à cette seconde opération. Reste à présent à examiner, sur la base même de cette définition de la santé mentale et de son lien avec la vie sociale, comment la notion de bon sens supérieur se trouve au cœur d'un renversement de la pensée bergsonienne prenant corps dans Les deux sources et qui a pour but de rendre possible l'action morale.

C'est parce que le bon sens est au cœur de la réflexion bergsonienne sur le rapport de l'individu aux normes sociales et sur la justice que l'étude de cette notion permet d'aborder sous un jour nouveau l'itinéraire et l'évolution de la pensée bergsonienne concernant l'action juste. Cette problématique, laissée de côté de Matière et Mémoire à l'Évolution créatrice, réapparaît dans Les deux sources et la plupart de ce qui était posé à titre de postulat dans le discours de 1895 y est repris, repensé et intégré à la problématique plus vaste d'une réflexion sur les rapports qui unissent morale et société. Le discours de 1895 apparaît donc comme un programme, un ensemble d'intuitions abandonnées en l'absence d'un appareillage conceptuel suffisant. Il est important de souligner les correspondances qui existent entre les deux œuvres pour mieux comprendre ce qui explique l'abandon puis la reprise de la problématique morale dans la définition de la notion de bon sens dans l'œuvre de Bergson. Ainsi, l'idée d'une action de bon sens orientée vers le progrès de l'ensemble de la société était soutenue dans le discours par le pressentiment d'un lien unissant principe de la vie et capacité à produire des actions justes et progressistes de la part de l'individu de bon sens. Bergson y affirmait en effet de la notion de bon sens : «si elle porte ainsi avec elle l'intelligence de la vie, c'est sans doute qu'elle en a touché le principe $»^{25}$, cette idée essentielle d'un fondement de la morale dans la prise de contact avec le principe de la vie se retrouve dans Les deux sources où Bergson évoque au sujet des agents du progrès humain l'action de «l'élan de la vie, cet élan même, communiqué intégralement à des privilégiés qui voudraient alors l'imprimer à l'humanité entière ${ }^{26}$. Et de fait, à l'idée exprimée dans le discours selon laquelle « il est rare que la nature produise spontanément une âme affranchie et maîtresse d'elle-même, une âme

24. H. Bergson, Le Rire, op. cit., p. 452-453.

25. Id., Le bon sens et les études classiques, op. cit., p. 365.

26. Les deux sources de la morale et de la religion, op. cit., p. 1174. 
accordée à l'unisson de la vie» ${ }^{27}$, répond l'affirmation des Deux sources selon laquelle les mystiques et les êtres novateurs constituent en eux-mêmes « une espèce nouvelle composée d'un individu unique ${ }^{28}$. En outre, la définition du bon sens comme «ignorance consciente d'elle-même ${ }^{29}$ dans le discours de 1895 trouve un écho dans la définition du bon sens supérieur donnée dans les Deux sources comme «innocence acquise», Bergson ajoutant dans les deux cas que cette ignorance doit s'accompagner d'un effort. Là n'est sans doute pas pourtant le plus important: en effet, la principale difficulté du discours est l'affirmation selon laquelle le bon sens produit irrémédiablement les actions les plus justes possibles ${ }^{30}$ dans un contexte donné, sans qu'il consiste pourtant «ni dans une expérience plus vaste, ni dans des souvenirs mieux classés, ni même, plus généralement, dans une logique plus rigoureuse ${ }^{31}$, arguant qu'il choisit la meilleure solution à une situation donnée parce qu'il peut en "prévoir [1]es conséquences, ou plutôt les pressentir ${ }^{32}$. La question de l'infaillibilité morale de l'action de bon sens est finalement abordée à nouveau par Bergson lorsqu'il évoque dans Les deux sources le bon sens supérieur des mystiques, en termes de «discernement prophétique du possible et de l'impossible $»^{33}$ qui fournit «du premier coup la démarche utile, l'acte décisif, le mot sans réplique $»^{34}$. De plus, on retrouve enfin clairement exprimée l'idée que le bon sens est, comme on pouvait le lire dans le discours, un instrument de progrès social $»^{35}$, puisque Bergson, qui fait du bon sens supérieur l'apanage des mystiques, gratifie ceux-ci d'une capacité essentielle à « pousser l'humanité en avant ${ }^{36}$.

Si Bergson, après le discours, avait écarté au profit d'une réflexion sur la santé mentale cette problématique de l'action morale qu'il retrouvera dans Les deux sources, c'est peut être à cause de l'insuffisance des outils conceptuels dont il disposait en matière d'analyse des rapports entre morale et société en 1895, dans la mesure où il n'avait pas analysé les liens que la société entretient avec le développement d'une morale. En effet, la réflexion sociologique qui sous-tend le discours de 1895 semble assez irréaliste: poussée jusqu'au bout, elle implique que, n'était le pouvoir pétrificateur de l'intelligence, l'ensemble de la société, guidé par le bon sens, agirait toujours non seulement de manière parfaitement adaptée à la situation présente mais

27. Le bon sens et les études classiques, op. cit., p. 366.

28. H. Bergson, Les deux sources de la morale et de la religion, op. cit., p. 1056.

29. Le bon sens et les études classiques, op. cit., p. 362.

30. Ce que Guy Lafrance appelle une «justice intuitive», cf. La philosophie sociale de Bergson, sources et interprétation, op. cit., p. 115 et suiv.

31. H. Bergson, Le bon sens et les études classiques, op. cit., p. 364.

32. Ibid., p. 362.

33. Les deux sources de la morale et de la religion, op. cit., p. 1169.

34. Ibid. p. 1172.

35. H. Bergson, Le bon sens et les études classiques, op. cit., p. 364.

36. Les deux sources de la morale et de la religion, p. 1017. 
encore toujours en vue du plus grand bien et du plus grand progrès social, étant constamment animé par l' «esprit de justice ${ }^{37}$. Cette conception optimiste qui attribue comme principe à la vie sociale ce même «esprit de justice », puisqu' on «ne peu[t] [s]e représenter ces volontés associées sans une fin dernière raisonnable ${ }^{38}$, a cédé le pas dans les œuvres ultérieures à une analyse de l'origine vitale de la vie sociale orientée vers la survie de l'espèce et qui détermine, par ses normes, l'appréhension du réel : il semble donc que ce soit une modification au sein même de la réflexion bergsonienne sur la société, ses normes, et le rapport des individus à ces normes, qui a dû présider au destin de la notion de bon sens dans son œuvre. Si le discours de 1895 distinguait en passant une «justice abstraite» d'une «justice incarnée dans l'homme juste ${ }^{39}$ (la première étant discréditée au profit de la seconde), pour pouvoir penser l'acte véritablement moral, cette distinction n'était pas assez précise, ne s'appuyant pas sur l'arrière-plan sociologique élaboré dans Les deux sources qui seul permet, en distinguant la société close de la société ouverte, de distinguer par là même deux véritables types de justices : une justice relative et une justice absolue. C'est cette distinction qui permet en effet de résoudre le paradoxe du bon sens comme étant à la fois un sens de l'adaptation au réel social mais aussi une aspiration innée au progrès au sein de chaque individu, puisqu'elle permet de lier la première tendance à la justice relative, celle dont le fondement est œil pour œil, dent pour dent et qui s'exprime dans la dimension close de la société (puisque la raison d'être de la morale close est, comme l'ont souligné certains, l'adaptation de l'individu ${ }^{40}$ ). La seconde est désormais liée à une justice absolue, qui fonde une morale de l'aspiration, elle-même visant l'avènement d'une société ouverte.

En outre, dans Les deux sources, Bergson expose une double conception du rapport de l'individu aux normes sociales : en ce qui concerne la société de type clos, les normes sociales agissent sur l'individu dans la mesure où elles poussent son moi profond à se solidifier en s'extériorisant, processus auquel, nous l'avons vu, le bon sens ordinaire n'est pas étranger, mais l'idée d'une société ouverte permet de briser le cercle dans lequel tournoie l'espèce humaine, prise dans la circularité close, en permettant de penser l'influence de l'individu d'exception, ayant presque par miracle échappé à l'obsession de l'utilité vitale, sur la société, par le biais de la création de valeurs morales. À un mouvement passif de réception et d'intégration des normes afin de bien s'insérer dans le réel, analysé dans le Rire et l'Évolution créatrice, peut succéder un mouvement de projection hors de soi qui modifie en retour le réel, mouvement requis par les thèses du discours de 1895. La distinction

37. H. Bergson, Le bon sens et les études classiques, op. cit., p. 364 : «Instrument, avant tout, de progrès social, il ne peut tirer sa force que du principe même de la vie sociale, l'esprit de justice ».

38. Ibid., p. 366.

39. Le bon sens et les études classiques, op. cit., p. 364.

40. Georges Mourélos, Bergson et les niveaux de réalité, Paris, PUF, 1964, p. 175-176. 
entre une société close et une société ouverte est ce qui permet à Bergson de comprendre à nouveau le bon sens comme une poussée vers le progrès moral guidée, comme le voulait le discours de 1895, par l'esprit de justice qui lui montre « les injustices à corriger et le bien à faire » ${ }^{41}$. En effet, le bon sens supérieur recherche non pas l'adaptation à des normes préexistantes en vue de favoriser la survie individuelle, mais bien une action en retour sur la société, l'établissement de normes radicalement nouvelles dont le principe est désormais le contraire de celui d'utilité, à savoir : la justice absolue fondée sur l'amour. Le bon sens supérieur, loin de recevoir passivement une stimulation à l'action émanant des impératifs vitaux et sociaux de la situation présente, loin de fournir seulement une réponse aux questions posées par son environnement, précède toute sollicitation extérieure et vise à imposer dans la réalité commune la réponse à une sollicitation émanant du fond de l'individu. Il est ainsi un «goût de l'action » mis en jeu lorsque le mystique choisit de diffuser son esprit dans la matérialité et est encore, à ce titre, comme le bon sens ordinaire, une capacité de «s'adapter et se réadapter ${ }^{42}$ à la mouvance du réel. Cependant sa visée n'est plus l'ajustement aux exigences vitales mais bien, comme le voulait le discours, la production de «la plus grande somme de bien ${ }^{43}$. Ce n'est qu'au prix de la transformation du bon sens en un bon sens supérieur, appuyé sur une justice absolue et non plus relative, et «supérieur » puisqu'entre temps le bon sens avait été défini comme santé mentale, que Bergson a pu en 1932 retrouver ce qu'il avait seulement supposé en 1895.

Si elle permet de valider, par-delà l'analyse du bon sens comme santé mentale, les conclusions du discours de 1895, la distinction entre bon sens ordinaire, conduite sociale normale, et bon sens supérieur, conduite morale modifiant les normes sociales, est aussi l'annonce d'un changement dans la pensée morale bergsonienne. En effet, à présent, seul un nombre restreint de personnalités géniales possède le pouvoir de faire progresser la société que le discours de 1895 attribuait, à travers le bon sens comme santé de l'esprit, à chaque individu, à quelques degrés près de dispositions ${ }^{44}$. Ainsi, l'humanité ordinaire est réduite, en ce qui concerne la véritable morale, à un rôle passif d'imitation des grands modèles ${ }^{45}$, imitation qui devient dès lors, pour elle, le fondement de l'action morale et juste pour qui est dépourvu de géniale créativité : cette passivité est tout juste corrigée par l'idée que cette imitation peut aussi se faire communication de l'élan et être l'occasion d'une éclosion puisqu'il peut «y avoir en nous un mystique qui sommeille et qui attend

41. H. Bergson, Le bon sens et les études classiques, op. cit., p. 365.

42. Id., Deux sources, op. cit., p. 1169.

43. Id., Le bon sens et les études classiques, op. cit., p. 361.

44. Ibid., p. 360 : «Aussi la tâche de l'éducateur consiste surtout, en pareille matière à conduire les uns par un artifice, là où d'autres sont tout de suite placés par nature ».

45. H. Bergson, Deux sources de la morale et de la religion, op. cit., p. 1004 : «Chacun de nous [...] s'est demandé ce que tel ou tel eût attendu de lui en pareille occasion ». 
seulement une occasion de se réveiller ${ }^{46}$. Si le bon sens du discours trouve sa formulation finale dans le bon sens supérieur, alors l'espoir en un homme nouveau est interdit, puisque seule une élite morale, celle des mystiques et des êtres dotés du bon sens supérieur, peut constituer une espèce nouvelle, chaque fois réduite à un individu unique.

Le postulat de l'infaillibilité du bon sens à toujours produire l'action la plus juste, repris du discours dans Les deux sources, demande à être interrogé, et la question du fondement de la justice de l'action se pose d'autant plus âprement que, dans Les deux sources, Bergson rejette fermement aussi bien la tentative kantienne de fonder la morale dans la logique (ou ce qu'il voit comme tel) ${ }^{47}$ que les théories de type platonicien faisant dériver la justice de l'idée de Bien. En tant qu'instance poussant à accomplir irrémédiablement l'action la plus juste, il pourrait rappeler l'eustochia aristotélicienne, ou la «justesse de coup d'œil ${ }^{48}$ porté sur la situation, qui se passe de raisonnement, cependant, le fait que Bergson refuse de faire dériver le bon sens de l'habitude ou de l'expérience contredit ce rapprochement. Notons en tout cas que le fondement de l'excellence de l'action de bon sens semble reposer, suivant un schéma tout aristotélicien, sur l'excellence de l'agent qui dispose d'un bon sens supérieur, qu'elle contribue à exprimer en retour. Dans la mesure même où l'aspiration essentiellement morale du mystique ou du héros précède sa tendance à accomplir des actions morales, et que la crise mystique n'est pas l'expression du bon sens supérieur mais ce qui précède son apparition, c'est en définitive de l'excellence morale ou aspiration à la justice absolue que dérive la justice de l'action réalisée par le bon sens supérieur. Or dans la mesure où le bon sens supérieur se caractérise par un certain détachement à l'égard des exigences biologiques égoïstement utilitaires à la fois vitales et sociales (dans leur dimension close), détachement lié au fait que, même si «nous trouvons [la société] présente en nous », «sa présence est plus ou moins marquée selon les individus $»^{49}$, l'apparition de l'aspiration morale est due, comme le souligne Bergson, à une sorte de hasard vital comparable à celui qui préside, dans le Rire, à l'apparition des artistes ${ }^{50}$. Si la justesse de son ajustement à la situation présente est encore le signe du lien qu'entretient le bon sens supérieur avec les exigences vitales circulaires de la survie en société close, la justice de ce qu'il vise à produire a partie liée avec le vital au sens de la constante marche de l'élan, procédant par bonds, vers la

46. Ibid., p. 1060.

47. Bergson fonde probablement son interprétation de la morale kantienne sur l'analyse de la rupture d'une promesse ou celle du suicide dans Les fondements de la métaphysique des mours et dans le chapitre premier de l'Analytique de la Critique de la raison pratique. Il critique Kant en affirmant que «jamais [...] on ne sacrifierait au seul besoin de cohérence logique son intérêt, sa passion. »(Les deux sources, op. cit., p. 994).

48. Cf. Aristote, Ethique à Nicomaque, traduction Jean Tricot, Paris, Vrin, 1997, p. 298.

49. H. Bergson, Deux sources de la morale et de la religion, op. cit., p. 987.

50. Id., Le Rire, op. cit., p. 461. 
création d'une société ouverte. C'est en cela que le bon sens supérieur est révélateur au sein de la pensée bergsonienne de ce qu'on pourrait appeler la « contradiction du vital », c'est-à-dire la coexistence en son sein de l'élan et de la retombée.

Cette contradiction logée au cœur de la distinction entre les deux bons sens se retrouve dans l'expression de «contradiction réalisée », employée par Bergson pour désigner la capacité des mystiques à « convertir en effort créateur cette chose créée qu'est une espèce, faire un mouvement de ce qui est par définition un arrêt $»^{51}$. Si l'aspiration morale, qui brise le cercle dans lequel tournent les individus et la société close, est encore une expression du vital, c'est que celui-ci a été redéfini entre l'Évolution créatrice et Les deux sources, passant d'un élan vers la vie sociale à un élan qui contient lui-même un germe moral, puisque selon le dernier ouvrage «toute morale [...] est d'essence biologique $»^{52}$ et que le contact avec le principe vital est devenu la seule source où «puiser la force d'aimer l'humanité ${ }^{53}$. Si l'effort qui préside à ce contact est décrit par Bergson comme effort en sens inverse de la nature, c'est dans la mesure où la natura naturans effectue sa poussée contre l'inertie de la natura naturata: ainsi, la morale de l'aspiration qui unit les individus d'un bon sens supérieur est dite rendre l'individu à sa destination naturelle en le rendant créateur, en accord avec l'univers dans sa dimension de "machine à faire des dieux ${ }^{54}$. Par conséquent, la distinction entre un bon sens supérieur et un bon sens ordinaire, tous les deux naturels en ces deux sens opposés, exprime la spécificité de l'espèce humaine en laquelle coexistent, en tant qu'elle est une espèce sociale, élan (tension vers la société ouverte à travers des personnalités géniales) et retombée (tendance à la clôture). C'est dans un rapport croisé entre le «moi » et la réalité extérieure et sociale que s'exprime l'entrecroisement de ces deux naturels : ainsi, le bon sens supérieur, qui favorise l'action en direction du progrès de tous, est naturel dans la mesure où il naît d'un contact avec la réalité mouvante qui fait le fond des choses et les couches profondes du moi, ce qu'il y a de plus personnel, tandis que le bon sens ordinaire, qui vise un but essentiellement individuel (l'adaptation en vue de la survie), est le produit d'une solidification naturelle du moi par le biais de normes et de processus d'adaptation essentiellement impersonnels exerçant une force d'inertie, ou plutôt entraînant dans un mouvement circulaire. Si la crise mystique qui précède l'apparition $\mathrm{du}$ bon sens supérieur n'est pas sans rappeler l'explosion créatrice du moi d'en bas, dans l'Essai, c'est peut-être parce qu'elle constitue la forme finale prise par l'acte libre dans la pensée bergsonienne, dans la mesure où elle est pensée à partir de la prise en compte de la pression fondamentale que la société exerce sur la constitution même de l'individu (à laquelle participe le bon sens ordinaire) qui n'avait pas

51. Id., Deux sources, p. 1174.

52. Ibid., p. 1061.

53. Ibid., p. 1021.

54. H. Bergson, Les deux sources de la morale et de la religion, op. cit., p. 1245. 
encore été analysée au moment de l'Essai ${ }^{55}$. Exprimant les deux sens de la nature en l'homme, la distinction entre bon sens ordinaire et bon sens supérieur répond finalement à la constatation de ce que «la morale comprend [...] deux parties distinctes, dont l'une a sa raison d'être dans la structure originelle de la société humaine et dont l'autre trouve son explication dans le principe explicatif de cette structure $»^{56}$.

Cette distinction permet également de résoudre le problème du rapport du bon sens à la pratique artistique : c'est le bon sens supérieur, qui résulte d'un contact avec le principe créateur de la vie et produit des actes moraux prenant à leur tour la forme de créations (à la fois de normes nouvelles et d'un nouveau moi), qui est « dans la vie pratique, ce que le génie est dans les sciences et les arts ${ }^{57}$ selon la formule du discours, et c'est le bon sens ordinaire qui, étant lié à la perception utilitaire de la réalité qu'il contribue à morceler avec souplesse, s'oppose à la pratique artistique comme au rêve, selon les analyses du Rire.

La question de savoir si la supériorité morale ne peut surgir que d'un effort dirigé contre le bon sens ordinaire, antérieurement défini comme état normal de l'esprit humain et santé psychologique, et ses préoccupations utilitaires, engage la viabilité de la morale bergsonienne. En effet, si la santé mentale d'un individu est liée à sa disponibilité passive à l'égard de l'environnement social et si le fondement de l'aspiration morale qui génère le bon sens supérieur est la libération à l'égard de l'utilité vitale et des normes sociales, alors apparait la possibilité d'un conflit sous-jacent dans la pensée bergsonienne entre les définitions de l'individu normal et de l'individu moral. En ce qui concerne la problématique de la santé mentale, le principal interlocuteur, ou adversaire, de Bergson est Pierre Janet qui a pour ambition, dans De l'angoisse à l'extase, de mettre à jour la racine pathologique du délire mystique qu'il apparente à un trouble psychasthénique. À ce titre, il note que les «croyances brutales» de sa patiente Madeleine, qu'il compare à Thérèse d'Avila, «présentent en maints endroits des contradictions et ne tiennent aucun compte des règles vulgaires du bon sens $»^{58}$, la principale caractéristique du discours mystique étant pour lui d'être «une pensée en régression, analogue à celle des petits enfants et des sauvages ${ }^{59}$. Pour lui répondre, Bergson tente d'abord de distinguer l'anormal de l'extraordinaire, puis finit par admettre qu'en tant que passage à l'extraordinaire (c'est-à-dire au dynamique et à l'ouvert), la violence même

55. Le bon sens supérieur est lié à la partie la plus intime de lui-même que l'individu retrouve quand «sa conscience, travaillant en profondeur, lui révèle, à mesure qu'il descend davantage, une personnalité de plus en plus originale, incommensurable avec les autres et d'ailleurs inexprimable » (Henri Bergson, Les deux sources de la morale et de la religion, op. cit., p. 986).

56. H. Bergson, Deux sources de la morale et de la religion, op. cit., p. 1020.

57. Id., Le bon sens et les études classiques, op. cit., p. 361.

58. P. Janet, De l'angoisse à l'extase, Paris, Félix Alcan, 1926, p. 459.

59. Ibid., p. 464. 
du processus de libération mystique peut générer l'apparition de symptômes anormaux (au sens de morbides) et «déranger les rapports habituels entre conscient et inconscient ${ }^{60}$. Il est intéressant de constater que c'est de la présence, chez les mystiques, du bon sens supérieur sous la forme d'un "goût de l'action", c'est-à-dire de ce par quoi le bon sens supérieur ressemble au bon sens ordinaire, que Bergson cherche à tirer argument pour justifier la santé mentale des mystiques contre Janet. Mais, comme le note celui-ci contre l'idée que la foisonnante activité des mystiques serait le signe de leur santé mentale : "l'état proprement psychasthénique n'est pas constant et un malade [...] peut être en dehors de ces crises assez actif et persévérant pour accomplir des œuvres intéressantes ${ }^{61}$. Mais l'originalité du bon sens supérieur est d'unir, dans le prolongement de la «crise même » au sens où l'entend Janet, l'ajustement de l'action au réel et la visée plus haute inspirée par l'amour. C'est pourquoi Bergson va plus loin dans sa réponse et avance que le bon sens supérieur est une "santé intellectuelle solidement assise, exceptionnelle, qui se reconnaît sans peine ${ }^{62}$. Et, en effet, si l'on considère que l'aspiration morale est une forme de santé dans la mesure où elle est contact avec l'élan vital, nature naturante supérieure à la circularité de la nature naturée, et, dans la mesure où cette santé est réservée à une élite, alors se comprend le fait qu'il existe une santé mentale (en un sens non métaphorique) se caractérisant par l'exception et non par la moyenne : cette santé est normale en un sens normatif sans être normal en un sens statistique. Mais si le bon sens supérieur est une «santé intellectuelle », de quel genre d'état mental s'agit-il ? L'intelligence à laquelle Bergson fait référence ici ne peut pas être celle qui soumet le réel à son découpage utilitaire, mais au contraire une intelligence qui parvient à retrouver, entre les lignes de ce morcellement, le réel mouvant.

L'équilibre des facultés qui caractérisait le bon sens ordinaire se transforme dans le bon sens supérieur en un «équilibre d'un autre genre » ${ }^{63}$ auquel Bergson fait allusion au début des Deux sources, sans le lier à la problématique du bon sens supérieur qui n'a pas encore été évoquée, ainsi que le suggère allusivement le début des Deux sources, précisant toutefois au sujet de l'effort qui permet de s'enfoncer ainsi en soi à la découverte de son moi : «s'il est possible, il est exceptionnel ${ }^{64}$. Il est possible d'avancer que cet équilibre d'un nouveau genre peut être lié à une forme de perception réelle quoiqu'anormale, justement parce qu'elle n'est pas contrainte par les exigences de l'action: «il doit y avoir, soit dans le corps, soit dans la conscience qu'il limite, des dispositifs spéciaux dont la fonction est d'écarter de la perception humaine les objets soustraits par leur nature à l'action de l'homme. Que ces mécanismes se dérangent, la porte qu'ils maintenaient

60. H. Bergson, Les deux sources, op. cit., p. 1170.

61. P. Janet, De l'angoisse à l'extase, op. cit., p. 461.

62. H. Bergson, Les deux sources de la morale et de la religion, op. cit., p. 1169.

63. Ibid., p. 986.

64. Ibid., p. 986. 
fermée s'entrouvre: quelque chose passe d'un «en dehors », qui est peut être un «au-delà». C'est de ces perceptions anormales que s'occupe la «science psychique ${ }^{65}$. Ce qui serait ordinairement jugé pathologique, à savoir ces «perceptions anormales », reçoit finalement une justification morale, puisque ces perceptions émanent de la santé supérieure que constitue l'équilibre supérieur de l'esprit mystique soustrait aux exigences d'utilité. Cela signifie non seulement que le normal psychologique (qui n'est que l'état moyen de l'esprit des individus rivés à leur intérêt) n'a pas à recevoir un statut normatif, mais surtout que le moral est une norme qui enjoint de dépasser purement et simplement l'équilibre normal de l'esprit, le réel se trouvant modifié à son tour parce qu'étendu à ce que les exigences vitales rejetaient.

L'anormal est peut-être même alors plus réel que le réel morcelé par les exigences vitales, auquel l'état normal de l'esprit donne accès, car «tout se passe comme si l'équilibre humain, comme si la normalité engendrée par cet équilibre cessait d'être viable ; à un niveau plus profond, il est perçu et vécu comme un déséquilibre en tant que son modus vivendi ne cesse de réprimer les forces créatrices au sein de l'individu ${ }^{66}$. Ériger l'anormal en norme morale permet donc à Bergson de modifier en retour la définition du normal par la mise en question de la validité de la perception normale (c'est-à-dire habituelle) elle-même, qui était au fondement à la fois de la définition de l'adaptation au réel, mais aussi du réel lui-même. Ce renversement illustre finalement pleinement la contradiction qu'on trouve au sein de l'œuvre bergsonienne entre le vital compris comme ensemble d'exigences d'utilité pour la survie individuelle et d'autre part comme élan créateur : le second l'emporte finalement sur le premier puisque l'aspiration morale contribue à défaire le découpage utilitaire du réel et donne ainsi accès à l'élan vital lui-même.

65. Ibid., p. 1243.

66. D. Lapoujade, «Sur un concept méconnu de Bergson: l'attachement à la vie », in Frédéric Worms (dir.), Annales bergsoniennes, tome 4, Paris, PUF, 2009, p. 689. 